\title{
On the ellipticity of galaxy clusters
}

\author{
P. Flin, J. Krywult, M. Biernacka \\ Institute of Physics, Pedagogical University, Kielce, Poland
}

\begin{abstract}
We analysed 246 scanned ACO clusters and we do not find any dependence of galaxy cluster ellipticity on redshift.
\end{abstract}

\section{Introduction}

The recent substantial evolution in the ellipticity of rich galaxy clusters was noted by Mellot, Chambers \& Miller (2001). This result has been supported by Plionis (2002). In particular, he argues that the ellipticity of ACO clusters is correlated with their redshifts. Using, among others, 309 ACO clusters taken from APM, for which about 180 have determined redshifts, he claimed the existence of a significant ellipticity - redshift dependence.

\section{Data}

Our sample consists of 246 ACO clusters. All data come from 48" Schmidt telescopes. Original plates were scanned at the Rome and Edinburgh Astronomical Observatories. The majority of galaxy catalogues were obtained from DSS using FOCAS packages. For this sample, visual verification of object classification was made.

\section{Method of analysis}

It is well known that ellipticity depends also on the distance from cluster centre (e.g. Carter \& Metcalfe 1980, Flin 1984, Trevese et al. 1992, Struble \& Ftaclas 1994). Therefore, we decided to check the investigated relation for various distances from cluster centres. The radius changes from 0.5 to $1.5 \mathrm{Mpc}\left(h=0.75, q_{0}=0.5\right)$ with a step of 0.25 $\mathrm{Mpc}$ from the cluster centre. Thus, we have measured ellipticities at 5 distances from the cluster centre for each of the 246 clusters. The ellipticities have been determined in a standard way, this is from the covariance matrix. The redshifts are taken from the literature.

\section{Results}

For each cluster we have two parameters, the calculated ellipticity of the cluster and cluster redshift. A straight line was fitted to each set of data. The results of the fitting procedure for the line $e=A+B \cdot z$ are given in Table 1 . The parameter $R$ in Mpc is the radius of the circle in which the ellipse has been calculated, $A$ and $B$ are the fit parameters and $r$ is the correlation coefficient. We also calculated the mean value of the five ellipticities for various $R$ radii. The dependence of such mean ellipticities on redshift is given in Fig.1. The last line of Table 1 gives the relevant parameters. 


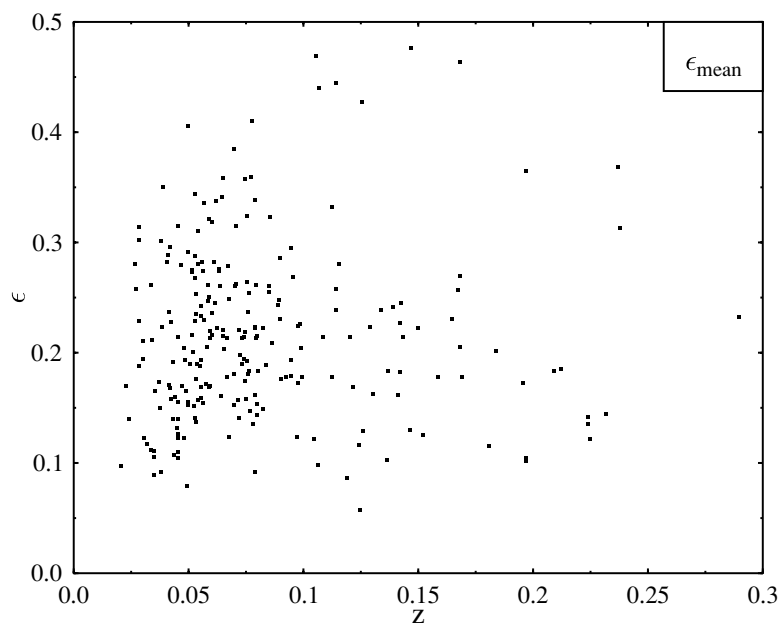

Figure 1. The relation between ellipticity and redshift for 246 investigated ACO clusters

Table 1. The result of the correlation analysis of the investigated samples

\begin{tabular}{ccccc}
\hline$N$ & $R$ & $A$ & $B$ & $r$ \\
\hline 1 & 0.50 & 0.298 & 0.218 & 0.05 \\
2 & 0.75 & 0.215 & 0.118 & 0.05 \\
3 & 1.00 & 0.207 & -0.097 & 0.05 \\
4 & 1.25 & 0.186 & -0.115 & 0.06 \\
5 & 1.50 & 0.176 & -0.061 & 0.04 \\
\hline 6 & Mean & 0.216 & 0.013 & 0.01 \\
\hline
\end{tabular}

\section{Conclusions}

It is clear that we do not observe any relation between ellipticities and redshifts. This differs greatly from the Plionis (2002) result. However, the claimed dependence of ellipticity on redshift reported by him is very weak. Our result is affected by such factors as e.g. the adopted cluster centre, the way of smoothing the distribution and the applied method of ellipticity determination. These factors are being carefully studied now. The current result is consistent with Mulchaey \& Zabludoff (1998) and Zabludoff \& Mulchaey (1998) observation reporting the lack of recent significant dynamical evolution for poor groups and $\mathrm{cD}$ clusters. Moreover, the lack of the eccentricity evolution is in agreement with the latest hydrodynamic simulations of clusters (Floor et al. 2003a, 2003b).

\section{Acknowledgements}

This paper was financially supported by Pedagogical University grants: BS 052, BW 116 and BW 236. 


\section{References}

Carter, D., Metcalfe, N. 1980 MNRAS, 191, 325

Flin, P. 1984 in Clusters and Groups of Galaxies, eds. Mardirossian, F., et al., Dordrecht, p. 163

Floor, S.N., Melott, A.L., Miller, C.J., Bryan, G.L. 2003a, ApJ, 591, 741

Floor, S.N., Melott, A.L., Motl, P.M. 2003b, astro-ph/0307539

Melott, A., Chambers, S. W., Miller, C. J. 2001, ApJ, 559, L75

Mulchaey, J. S., Zabludoff, A. I. 1998, ApJ, 496, 73

Plionis, M. 2002, ApJL, 572, 67

Struble, M. C., Ftaclas, F. 1994, AJ, 108, 1

Trevese, D., Flin, P., Migliori, L., Hickson, P., Pittella, G. 1992, A\&AS, 94, 237

Zabludoff, A. I., Mulchaey, J. S. 1998, ApJ, 496, 39 